\section{Composição dos gastos privados com medicamentos utilizados por aposentados e pensionistas com idade igual ou superior a 60 anos em Belo Horizonte, Minas Gerais, Brasil}

\author{
Out-of-pocket drug expenditures by retirees and \\ pensioners 60 years and older in Belo Horizonte, \\ Minas Gerais, Brazil
}

Marina Guimarães Lima ${ }^{1}$ Andréia Queiroz Ribeiro 1 Francisco de Assis Acurcio 1 Suely Rozenfeld 2

Carlos Henrique Klein 2

\footnotetext{
1 Faculdade de Farmácia, Universidade Federal de Minas Gerais,

Belo Horizonte, Brasil. 2 Escola Nacional de Saúde Pública Sergio Arouca, Fundação Oswaldo Cruz, Rio de Janeiro, Brasil.

Correspondência M. G. Lima

Departamento de Farmácia Social, Faculdade de Farmácia, Universidade Federal de Minas Gerais. Rua Peru 111, apto. 204-Sion, Belo Horizonte, $M G$ 30320-040, Brasil. marina.glima@ig.com.br
}

\begin{abstract}
The aim of this study was to analyze the structure of private drug expenditures by individuals 60 years and older in Belo Horizonte, Minas Gerais, Brazil. The study population consisted of a representative sample of retirees under the National Social Security Institute (INSS) in the city of Belo Horizonte, interviewed through a household survey. Monthly out-of-pocket drug expenditures were calculated, and a drug cost structure analysis was performed according to drug characteristics. 667 elders answered the survey. Mean drug expenditures per month were US\$38.91. The therapeutic groups representing the majority of drug expenditures were: cardiovascular system (26\%), nervous system (24\%), and digestive/metabolic system (15\%). Considering drug registration categories, brand-name drugs accounted for the majority of expenditures (54\%). The results of this study can support policies to improve both access to medicines and overall health conditions for the Brazilian elderly population.
\end{abstract}

Health of the Elderly; Health Economics; Drug Costs; Pharmacoepidemiology

\section{Introdução}

A proporção de idosos na população brasileira vem aumentando rapidamente 1,2 . O envelhecimento populacional demanda um planejamento dos sistemas de saúde para atender às necessidades específicas dos idosos. Segundo informações da Pesquisa Nacional por Amostra de Domicílios realizada em 1998 (PNAD 1998), 69\% dos idosos brasileiros relataram ter pelo menos uma doença crônica 3 .

Em acréscimo à prevalência das doenças crônicas, a população idosa também é caracterizada pela alta utilização de medicamentos. A maioria dos estudos de utilização de medicamentos relata o uso de um número médio de quatro medicamentos por idoso 4,5,6,7,8,9. O perfil de utilização de medicamentos por idosos acarreta em gasto elevado com a aquisição dos mesmos em comparação ao observado nas demais faixas etárias 10,11.

Os gastos com medicamentos utilizados pelos idosos são concentrados em determinadas categorias terapêuticas. Considerando o primeiro nível de classificação Anatomical Therapeutical Chemical (ATC) 12 dos medicamentos, as categorias sistema cardiovascular, sistema nervoso e trato alimentar e metabolismo são responsáveis pela maior parte dos gastos por idosos 13,14,15. A concentração dos gastos também é observada em relação à categoria e ao tempo de registro dos medicamentos. Em um estudo realizado na po- 
pulação idosa nos Estados Unidos, foi observado que os medicamentos novos representaram $20 \%$ dos medicamentos utilizados e $30 \%$ dos gastos com medicamentos, enquanto os medicamentos genéricos representaram $34 \%$ dos medicamentos utilizados pelos idosos e $11 \%$ dos gastos 14 .

No Brasil, segundo a PNAD 1998, o gasto médio com medicamentos referidos pelos idosos foi igual a $23 \%$ do valor do salário mínimo ${ }^{3}$. Entretanto, não há informações sobre a composição dos gastos pelos idosos brasileiros, pois não foram coletados dados sobre os medicamentos utilizados pelos mesmos na PNAD 1998.

No presente trabalho foram analisados os gastos mensais com medicamentos utilizados por indivíduos com 60 anos ou mais de idade em Belo Horizonte, Minas Gerais, Brasil, com ênfase nos gastos privados, considerando a composição dos mesmos segundo as características dos medicamentos.

\section{Métodos}

O presente trabalho constitui um estudo de custos baseado nas informações do inquérito populacional Perfil de Utilização de Medicamentos por Aposentados Brasileiros. Os métodos empregados nesse inquérito encontram-se descritos detalhadamente em uma outra publicação 16 .

Os participantes do inquérito foram selecionados aleatoriamente com base no cadastro de aposentados e pensionistas do Ministério da Previdência e Assistência Social, de forma a constituírem uma amostra representativa dos aposentados e pensionistas residentes em Belo Horizonte com idade igual ou superior a 60 anos. Os indivíduos selecionados foram entrevistados no domicílio, no período de março a junho de 2003. Na entrevista, foi utilizado um questionário para coletar informações sobre as características sócio-demográficas do participante, condições de saúde e uso de serviços de saúde, e utilização de medicamentos nos últimos 15 dias.

Os medicamentos utilizados pelos participantes do inquérito domiciliar foram classificados segundo o setor de aquisição, a categoria terapêutica, a categoria e o tempo de registro na Agência Nacional deVigilânciaSanitária (ANVISA) e a essencialidade. Os medicamentos magistrais foram excluídos da presente análise. Os setores de aquisição considerados foram: o setor público e o privado. As categorias terapêuticas consideradas na classificação dos medicamentos foram as presentes no ATC 12. Foram considerados dois níveis do sistema de classificação ATC dos medicamentos: o primeiro, que representa os grupos anatômicos, e o terceiro, que considera os sub- grupos farmacológicos. As categorias de registro dos medicamentos na ANVISA consideradas foram medicamentos de referência, similares e genéricos 17 . Em relação ao tempo de registro, foram considerados como medicamentos novos os registrados na ANVISA nos cinco anos anteriores à realização da entrevista, ou seja, de 1998 a 2003. Nos cinco primeiros anos de comercialização do medicamento, o mesmo é caracterizado como novo e é requerido um alerta sobre os seus possíveis riscos em sua bula 18 . A essencialidade do medicamento foi definida como a presença do mesmo na Relação Nacional de Medicamentos Essenciais (RENAME) 19. Conceitualmente, os medicamentos essenciais são os destinados a atender às necessidades da maioria da população, devendo ser eficazes e seguros e disponibilizados de forma contínua 20 .

Os preços dos medicamentos industrializados obtidos no setor privado foram coletados com base nos preços máximos ao consumidor permitidos pela legislação ${ }^{21}$. A fonte de pesquisa dos preços dos medicamentos foi a Revista ABCFarma (vol. 11, $\mathrm{n}^{\mathrm{o}}$. 139) do mês de março de 2003. Para refletir os impostos incidentes sobre os medicamentos no Estado de Minas Gerais, os preços considerados no cálculo dos gastos com medicamentos genéricos foram os preços ao consumidor com Imposto sobre Circulação de Mercadorias e Serviços (ICMS) de $12 \%$, e com os outros medicamentos foram os preços ao consumidor com ICMS de $18 \% 22$.

$\mathrm{O}$ cálculo dos gastos com os medicamentos obtidos no setor público foi realizado a partir dos preços de aquisição destes por instituições governamentais responsáveis pelo fornecimento de medicamentos para as unidades de saúde do Sistema Único de Saúde (SUS) em Belo Horizonte. A partir das informações referentes ao princípio ativo, apresentação farmacêutica e laboratório fabricante do medicamento, estimou-se a instituição pública que o adquiriu no ano de 2003, dentre as seguintes: Secretaria Municipal de Saúde de Belo Horizonte, Secretaria de Estado de Saúde de Minas Gerais e o Ministério da Saúde.

A estimativa dos gastos mensais privados com os medicamentos utilizados pelos indivíduos com 60 anos ou mais de idade foi realizada considerando o preço da unidade posológica de cada medicamento e a posologia padrão do mesmo em sua indicação principal.

Os gastos mensais privados com medicamentos foram expressos em duas unidades monetárias correntes: a brasileira, Real (R\$), e a estadunidense, Dólar (US\$). Foi considerada a cotação média do Dólar do período da realização da entrevista domiciliar, que foi de março a junho de 2003 (Banco Central do Brasil. Cotações 
e boletins. http://www.bcb.gov.br, acessado em 21/Jul/2005). Os gastos também foram expressos em proporções do salário mínimo vigente no período de $1^{\circ}$ de abril de 2003 a 30 de abril de 2004, de $\mathrm{R} \$ 240,0023$.

Os gastos mensais privados com os medicamentos industrializados utilizados pelos participantes do estudo foram descritos segundo a sua composição, considerando as categorias terapêuticas ATC, a categoria e tempo de registro e a essencialidade dos mesmos. Na descrição dos gastos segundo o primeiro nível de classificação ATC dos medicamentos, o medicamento foi considerado como unidade de análise. Na descrição dos gastos segundo o terceiro nível de classificação ATC, a categoria e o tempo de registro e a essencialidade dos medicamentos, foram considerados como unidades de análise os indivíduos que utilizaram pelo menos um medicamento de cada categoria. Os gastos foram descritos por meio de medidas de tendência central (média e mediana) e de dispersão (desvio-padrão). A análise estatística foi realizada empregando-se o programa SPSS 11.5 (SPSS Inc., Chicago, Estados Unidos).

\section{Resultados}

\section{Descrição da população estudada}

Dentre os 881 indivíduos selecionados para o inquérito domiciliar em Belo Horizonte, 667 concordaram em ser entrevistados e responderam aos questionários.

Observou-se uma predominância do sexo feminino na população entrevistada, correspondendo a $64 \%$ dos indivíduos. A idade dos participantes variou de 60 a 101 anos, com uma média de 71,4 e uma mediana de 70 anos de idade.

A população entrevistada também se caracterizou pela baixa escolaridade, com $45 \%$ dos participantes com curso primário incompleto. O valor médio dos benefícios recebidos mensalmente do INSS foi R $\$ 403,71$ (US\$ 127,76) e a mediana R\$ 200,00 (US\$ 63,29).

\section{Utilização de medicamentos}

Dentre os 667 indivíduos com 60 anos ou mais de idade que participaram do inquérito domiciliar em Belo Horizonte, 590 (88\%) utilizaram medicamentos nos últimos 15 dias. Foi observado um total de 2.478 medicamentos utilizados pelos 590 participantes. O número de medicamentos utilizados pelos mesmos variou de 1 a 19, com uma média de 4,2 e uma mediana de 4,0 medicamentos por indivíduo. Observou-se que 527 indivíduos utilizaram pelo menos um medicamento adquirido no setor privado e 233 utilizaram pelo menos um medicamento adquirido no setor público. Os medicamentos adquiridos no setor privado representaram $75 \%$ dos medicamentos utilizados. As categorias terapêuticas ATC no primeiro nível de classificação dos medicamentos mais freqüentemente utilizadas no setor privado foram o sistema cardiovascular (27\%), sistema nervoso (20\%) e trato alimentar e metabolismo (14\%). Em relação à categoria de registro dos medicamentos obtidos no setor privado, $49 \%$ eram similares, $6 \%$ eram genéricos e $45 \%$ eram medicamentos de referência. Cerca de $27 \%$ dos medicamentos adquiridos no setor privado foram considerados essenciais.

\section{Composição geral dos gastos com medicamentos}

Os gastos mensais totais com os 1.822 medicamentos utilizados por 527 participantes e adquiridos no setor privado atingiram $\mathrm{R} \$ 64.805,11$, variando de $R$ \$ 0,39 a $R$ \$ 1.528,02. A cotação do Dólar considerada na análise dos gastos foi a média das cotações do período da realização da entrevista domiciliar, que foi de março a junho de 2003. O valor médio cotado foi $\mathrm{R} \$ 3,16$. O gasto mensal médio individual no setor privado foi R\$ 122,97 (US\$ 38,91), com uma mediana de $\mathrm{R} \$ 69,91$ (US\$ 22,12) (Tabela 1). Esses valores estimados de gastos mensais com medicamentos equivalem, respectivamente, a $51 \%$ e a $29 \%$ do valor do salário mínimo vigente à época da realização das entrevistas.

Os gastos privados com os medicamentos utilizados pelos indivíduos com 60 anos ou mais de idade também foram caracterizados pela concentração. Os dez indivíduos com maiores gastos foram responsáveis por $17 \%$ dos gastos totais.

Já os gastos mensais totais com os 595 medicamentos obtidos no setor público e utilizados por 233 indivíduos foram de $\mathrm{R} \$ 3.255,82$, o que representou um gasto público médio de $\mathrm{R} \$ 13,97$ por indivíduo, com uma mediana de $\mathrm{R} \$ 3,36$.
Tabela 1

Gastos mensais privados com medicamentos utilizados pelos aposentados e pensionistas com 60 anos ou mais de idade. Belo Horizonte, Minas Gerais, Brasil, 2003 ( $n=527$ ).

\begin{tabular}{lcc}
\hline Unidade do gasto & Gasto médio \pm DP & Gasto mediano \\
\hline Real (R\$) & $122,97 \pm 182,34$ & 69,91 \\
Dólar (US\$) & $38,91 \pm 57,70$ & 22,12 \\
\hline
\end{tabular}


Composição dos gastos privados com medicamentos por categorias terapêuticas

Os grupos terapêuticos segundo o primeiro nível de classificação ATC que representaram uma maior proporção dos gastos mensais no setor privado foram, nesta ordem: sistema cardiovascular, sistema nervoso e trato alimentar e metabolismo (Tabela 2).

A Tabela 3 mostra os dez grupos terapêuticos com maiores gastos mensais segundo o terceiro nível de classificação ATC e os gastos efetuados pelos 269 indivíduos que utilizaram pelo menos um medicamento dessas categorias.

\section{Composição dos gastos privados com medicamentos por categoria e tempo de registro}

Em relação à categoria de registro dos medicamentos utilizados pelos participantes, 53,6\% dos gastos mensais privados totais foram efetuados com os medicamentos de referência, $41,7 \%$ com os similares e $4,7 \%$ com os genéricos.

Os gastos mensais privados pelos indivíduos que utilizaram pelo menos um medicamento de cada categoria de registro encontram-se descritos na Tabela 4.

Os gastos mensais com medicamentos novos representaram $16 \%$ dos gastos privados totais, apesar deste grupo ter representado 5\% dos medicamentos utilizados pelos indivíduos com 60 anos ou mais de idade. O gasto mensal privado médio com os medicamentos novos foi de R\$ 102,22 (US\$ 32,35), considerando os 104 indivíduos que utilizaram pelo menos um medicamento novo.

\section{Composição dos gastos privados com medicamentos por essencialidade}

Os medicamentos essenciais representaram 19\% dos gastos mensais privados, apesar de terem representado $27 \%$ dos medicamentos utilizados pelos indivíduos com 60 anos ou mais de idade. Os gastos mensais médio e mediano com os medicamentos essenciais foram R $\$ 39,81$ (US\$ 12,60 ) e R $\$ 25,17$ (US\$ 7,96), respectivamente. Em contraste, os gastos mensais médio e mediano com os medicamentos não-essenciais foram R \$ 109,03 (US\$ 34,50) e R \$ 53,77 (US\$ 17,02), respectivamente.

\section{Discussão}

Os gastos mensais privados com medicamentos utilizados pelos participantes do inquérito residentes em Belo Horizonte apresentaram-se elevados, considerando que a média de valores de benefícios do INSS recebidos mensalmente pelos mesmos foi $\mathrm{R} \$ 403,71$ e que cerca de $72 \%$ recebiam até dois salários mínimos. Entretanto, participantes do estudo poderiam ter utilizado uma

Comparação entre freqüência de utilização e composição dos gastos mensais privados totais com medicamentos, segundo os grupos anatômicos e terapêuticos no primeiro nível de classificação Anatomical Therapeutical Chemical (ATC) 12. Belo Horizonte, Minas Gerais, Brasil, 2003 ( $n=1.822$ ).

\begin{tabular}{lcc}
\hline Grupos anatômicos e terapêuticos & Freqüência de utilização & Percentual dos gastos \\
\hline Sistema cardiovascular & 26,8 & 26,4 \\
Sistema nervoso & 20,2 & 24,3 \\
Trato alimentar e metabolismo & 14,4 & 14,7 \\
Agentes de ação no sistema músculo-esquelético & 7,2 & 11,8 \\
Sistema respiratório & 6,3 & 3,0 \\
Órgãos sensoriais & 5,7 & 3,2 \\
Sangue e órgãos formadores de sangue & 5,5 & 3,6 \\
Agentes dermatológicos & 4,6 & 2,3 \\
Sistema geniturinário e hormônios sexuais & 3,1 & 3,8 \\
Preparações hormonais sistêmicas & 2,5 & 1,2 \\
Antiinfecciosos gerais para uso sistêmico & 2,0 & 2,6 \\
Agentes antineoplásicos e imunomoduladores & 0,2 & 1,1 \\
Agentes antiparasitários & 0,1 & $<0,05$ \\
Sem classificação & 1,4 & 2,0 \\
\hline
\end{tabular}


Gastos mensais privados com medicamentos industrializados, segundo os grupos terapêuticos no terceiro nível de classificação Anatomical Therapeutical Chemical (ATC) 12. Belo Horizonte, Minas Gerais, Brasil, 2003 ( $\mathrm{n}=269$ ).

\begin{tabular}{|c|c|c|c|c|c|}
\hline \multirow[t]{2}{*}{ Dez grupos terapêuticos de maior gasto } & \multirow[t]{2}{*}{$\mathbf{N}$} & \multicolumn{2}{|c|}{ Gasto em Reais (R\$) } & \multicolumn{2}{|c|}{ Gasto em Dólar (US\$) } \\
\hline & & Média \pm DP & Mediana & Média \pm DP & Mediana \\
\hline Medicamentos que afetam a estrutura óssea e a mineralização & 5 & $817,99 \pm 449,52$ & 887,48 & $258,86 \pm 142,25$ & 280,85 \\
\hline Medicamentos antidemência & 15 & $231,23 \pm 209,11$ & 109,13 & $73,17 \pm 66,17$ & 34,53 \\
\hline Antidepressivos & 37 & $83,91 \pm 48,57$ & 62,62 & $26,55 \pm 15,37$ & 19,82 \\
\hline Medicamentos antiulcerosos & 39 & $83,76 \pm 102,75$ & 59,77 & $26,50 \pm 32,52$ & 18,92 \\
\hline Agentes redutores de colesterol e triglicerídeos & 41 & $75,63 \pm 31,28$ & 74,21 & $23,93 \pm 9,90$ & 23,48 \\
\hline Hipoglicemiantes orais & 36 & $53,57 \pm 74,53$ & 33,53 & $16,95 \pm 25,58$ & 10,61 \\
\hline Antiinflamatórios e anti-reumáticos não-esteróides & 69 & $38,37 \pm 42,58$ & 18,23 & $12,14 \pm 13,48$ & 5,77 \\
\hline Ansiolíticos & 54 & $36,86 \pm 24,46$ & 36,63 & $11,67 \pm 7,74$ & 11,59 \\
\hline Inibidores da enzima conversora de angiotensina & 81 & $26,43 \pm 13,17$ & 23,17 & $8,36 \pm 4,17$ & 7,33 \\
\hline Agentes beta-bloqueadores associados a outros diuréticos & 8 & $25,71 \pm 11,30$ & 21,29 & $8,13 \pm 3,58$ & 6,74 \\
\hline
\end{tabular}

Tabela 4

Gastos mensais privados com medicamentos utilizados pelos aposentados e pensionistas com 60 anos ou mais de idade, segundo as categorias de registro. Belo Horizonte, Minas Gerais, Brasil, 2003 ( $n=527$ ).

\begin{tabular}{|c|c|c|c|c|c|}
\hline \multirow[t]{2}{*}{ Categorias de registro } & \multirow[t]{2}{*}{$\mathbf{N}$} & \multicolumn{2}{|c|}{ Gastos em Reais (R\$) } & \multicolumn{2}{|c|}{ Gastos em Dólar (US\$) } \\
\hline & & Média \pm DP & Mediana & Média \pm DP & Mediana \\
\hline Similar & 424 & $63,77 \pm 132,29$ & 27,62 & $20,18 \pm 41,86$ & 8,74 \\
\hline Genérico & 95 & $31,88 \pm 26,91$ & 22,67 & $10,09 \pm 8,51$ & 7,05 \\
\hline Referência & 392 & $88,61 \pm 139,52$ & 42,25 & $28,04 \pm 44,15$ & 13,37 \\
\hline
\end{tabular}

quantidade de medicamentos inferior à necessária devido a restrições orçamentárias para adquiri-los. Essa situação pode ter superestimado o cálculo dos gastos com medicamentos, assim como a eventual aquisição dos medicamentos a preços inferiores aos preços máximos autorizados pela legislação vigente, o que é freqüente no Município de Belo Horizonte. Essa estratégia de venda das grandes redes no comércio varejista farmacêutico, que oferecem descontos nos preços de determinados medicamentos para aumentar a demanda pelos mesmos, não pôde ser considerada no cálculo global dos gastos.

A proporção dos gastos com medicamentos utilizados pelos indivíduos com 60 anos ou mais de idade nos gastos totais em determinadas categorias terapêuticas apresentou-se superior à proporção que estas categorias representaram no total de medicamentos utilizados, como por exemplo nas categorias terapêuticas no primeiro nível de classificação ATC: sistema nervoso e sistema músculo-esquelético. Esses gastos podem ter ocorrido devido ao custo de tratamento elevado com os medicamentos nas seguintes categorias terapêuticas no terceiro nível de classificação ATC: agentes que afetam a estrutura óssea e a mineralização; antidepressivos e; medicamentos antidemência. Vale ressaltar que essas três categorias terapêuticas são destinadas ao tratamento de condições prevalentes em idosos, como osteoporose, depressão e déficit cognitivo. São necessários estudos na população idosa brasileira para determinar se os benefícios proporcionados pelos medicamentos dessas categorias terapêuticas são proporcionais aos custos de tratamento elevados dos mesmos. Segundo alguns estudos internacionais de avaliação econômica, esses medicamentos apresentaram-se custo-efetivos 24,25 , porém os resultados de outro estudo demonstraram que os medicamentos antidemência não foram custo-efetivos no tratamento da doença de Alzheimer 26. 
Foi observado no presente estudo uma baixa proporção de medicamentos genéricos nos gastos privados com medicamentos (5\%), que pode ter ocorrido devido à baixa participação dos mesmos no mercado farmacêutico brasileiro no início de 2003. Em janeiro desse ano, o faturamento com os medicamentos genéricos representava $6,6 \%$ do mercado 27 . A utilização dos genéricos pela população brasileira pode aumentar com a expansão desse mercado. O mercado dos medicamentos genéricos vem apresentando um crescimento anual médio de $11 \%$, representando $8,9 \%$ do faturamento do mercado farmacêutico brasileiro em maio de 2005 28. Nos Estados Unidos, onde a disponibilidade de medicamentos genéricos é superior e por um maior período de tempo, foi observada uma maior utilização dos mesmos por idosos. Segundo um estudo sobre gastos com medicamentos por idosos cobertos por um sistema de benefícios farmacêuticos nos Estados Unidos, os genéricos representaram 34\% dos medicamentos e $11 \%$ dos gastos 14 . O uso de medicamentos genéricos pode ser uma estratégia para o aumento da eficiência dos gastos com medicamentos por idosos, pois proporcionam os mesmos benefícios clínicos dos medicamentos de referência e são comercializados a preços inferiores.

Os medicamentos novos representaram $16 \%$ dos gastos privados mensais com medicamentos, apesar de terem representado apenas $5 \%$ dos medicamentos utilizados pelos participantes do presente estudo. Essa situação pode ter ocorrido devido ao preço elevado dos medicamentos novos, protegidos por patentes na maior parte dos casos. Além de comprometer o orçamento pessoal dos idosos, o uso de medicamentos novos pode acarretar riscos à saúde dos mesmos. A informação sobre a eficácia e segurança dos medicamentos novos é limitada, principalmente considerando que os indivíduos idosos geralmente não são incluídos nos ensaios clínicos 29 .
O uso de medicamentos essenciais representou uma menor proporção dos gastos privados totais (19\%) em comparação à proporção no conjunto dos medicamentos utilizados (27\%). Os gastos mensais com os medicamentos essenciais foram inferiores aos gastos com medicamentos não-essenciais. Esses resultados sugerem que o uso de medicamentos essenciais pode aumentar a eficiência dos gastos com medicamentos por idosos. Os medicamentos essenciais são preconizados pela Organização Mundial da Saúde (OMS) e reconhecidos como um dos elementos de melhor custo-benefício e grande impacto potencial na atenção à saúde 30 . Resultados de um estudo recente enfocando a disponibilidade de medicamentos em Minas Gerais enfatizaram a necessidade de divulgação e implementação do conceito de essencialidade no país 31 .

Ainda que os gastos públicos não tenham sido o enfoque principal deste trabalho, constatouse que o gasto médio (e mediano) individual no setor privado foi bastante superior ao observado no setor público. Entretanto não seria adequado comparar diretamente os gastos realizados pelos indivíduos com os gastos realizados pelo setor público, em função das especificidades dos mecanismos de aquisição praticados por esse setor. A importância da oferta pública no acesso aos medicamentos essenciais aponta para a necessidade de uma análise detalhada abordando os gastos públicos, o que extrapola os objetivos do presente trabalho.

Concluindo, os resultados observados no presente estudo podem ser úteis para subsidiar o planejamento de ações de saúde destinadas a melhorar o acesso a medicamentos e às condições sanitárias da população idosa brasileira. Assim, por exemplo, a promoção do uso de medicamentos essenciais, bem como a de genéricos, configuram estratégias importantes para aumentar a eficiência dos gastos, ademais de contribuir para incrementar a racionalidade do uso de medicamentos por esse grupo populacional. 


\section{Resumo}

O objetivo do presente estudo foi analisar a composição dos gastos privados com medicamentos utilizados por indivíduos com 60 anos ou mais de idade, em Belo Horizonte, Minas Gerais, Brasil. A população estudada foi uma amostra representativa de aposentados $e$ pensionistas do Instituto Nacional do Seguro Social (INSS) nessa faixa etária e residentes no Município de Belo Horizonte, entrevistados em um inquérito domiciliar. Foram calculados os gastos mensais com medicamentos obtidos no setor privado e analisada a sua composição considerando as características dos medicamentos. Responderam ao inquérito 667 indivíduos. Foi observado um gasto mensal privado médio de $R \$ 122,97$ (US\$38,91) com os medicamentos utilizados pelos participantes. Os grupos terapêticos que representaram uma maior proporção dos gastos totais foram: sistema cardiovascular (26\%), sistema nervoso (24\%) e trato alimentar e metabolismo (15\%). Em relação à categoria de registro dos medicamentos utilizados, os medicamentos de referência foram responsáveis por uma maior proporção dos gastos totais (54\%). Os resultados deste estudo podem subsidiar políticas destinadas a melhorar o acesso a medicamentos e às condições sanitárias da população idosa brasileira.

Saúde do Idoso; Economia da Saúde; Custos de Medicamentos; Farmacoepidemiologia

\section{Referências}

1. Instituto Brasileiro de Geografia e Estatística. Perfil dos idosos responsáveis pelos domicílios no Brasil 2000. Rio de Janeiro: Instituto Brasileiro de Geografia e Estatística; 2002. (Série Estudos e Pesquisas: Informação Demográfica e Socioeconômica, 9).

2. Kinsella K. Dimensiones demográficas y de salud en América Latina y el Caribe. In: Pérez EA, editor. La atención de los ancianos: un desafío para los anos noventa. Washington DC: Organización Panamericana de la Salud/Organización Mundial de la Salud; 1994. p. 3-18.

3. Lima-Costa MF, Barreto SM, Giatti L. Condições de saúde, capacidade funcional, uso de serviços de saúde e gastos com medicamentos da população idosa brasileira: um estudo descritivo baseado na Pesquisa Nacional por Amostra de Domicílios. Cad Saúde Pública 2003; 19:735-43.

4. Anderson G, Kerluke K. Distribution of prescription drug exposures in the elderly: description and implications. J Clin Epidemiol 1996; 49:929-35.

\section{Colaboradores}

M. G. Lima realizou a análise dos dados e foi a redatora principal do artigo. A. Q. Ribeiro, F. A. Acurcio, S. Rozenfeld e C. H. Klein participaram do desenvolvimento e execução do inquérito populacional no qual se baseou o estudo e colaboraram na análise dos dados e na redação do artigo.

\section{Agradecimentos}

A Cristiano Soares de Moura pela participação no desenvolvimento dos bancos de dados. Este estudo foi financiado pelo Ministério da Saúde do Brasil/Fundo Nacional de Saúde, por meio da então Gerência Técnica de Assistência Farmacêutica (GTAF/DAB/SPS - convênio $\mathrm{n}^{\circ}$. 796/2002), e também recebeu apoio logístico da Faculdade de Farmácia, Universidade Federal de Minas Gerais; da Escola Nacional de Saúde Pública Sergio Arouca; e da Casa de Oswaldo Cruz, ambas da Fundação Oswaldo Cruz.
5. Chrischilles EA, Foley DJ, Wallace RB, Lemke JH, Semla TP, Hanlon JT, et al. Use of medications by persons 65 and over: data from the established populations for epidemiologic studies of the elderly. J Gerontol 1992; 47:M137-44.

6. Coelho Filho JM. Perfil de utilização de medicamentos por idosos em área urbana do Nordeste do Brasil. Rev Saúde Pública 2004; 38:557-64.

7. Laukkanen P, Heikkinen E, Kauppinen M, Kallinen M. Use of drugs by non-institutionalized urban Finns born in 1904-1923 and the association of drug use with mood and self-rated health. Age Ageing 1992; 21:343-52.

8. Stuck AE, Beers MH, Steiner A, Aronow H, Rubenstein LZ, Beck JC. Inappropriate medication use in community-residing older persons. Arch Intern Med 1994; 154:2195-200.

9. Mosegui GBG, Rozenfeld S, Veras RP, Vianna CMM. Avaliação da qualidade do uso de medicamentos em idosos. Rev Saúde Pública 1999; 33:437-44. 
10. Metge C, Black C, Peterson S, Kozyrskyj AL. The population's use of pharmaceuticals. Med Care 1999; 37(6 Suppl):JS42-59.

11. Mueller C, Schur C, O'Connell J. Prescription drug spending: the impact of age and chronic disease status. Am J Public Health 1997; 87:1626-9.

12. World Health Organization Collaborating Centre for Drug Statistics Methodology. Anatomical Therapeutic Chemical (ATC) index with Defined Daily Doses (DDDs). http://www.whocc.no/atcddd (acessado em 15/Jan/2005)

13. Avery AJ, Groom LM, Brown KP, Thornhill K, Boot D. The impact of nursing home patients on prescribing costs in general practice. J Clin Pharm Ther 1999; 24:357-63.

14. Thomas CP, Ritter G, Wallack SS. Growth in prescription drug spending among insured elders. Health Aff (Millwood) 2001; 20:265-77.

15. Morgan SG, Agnew JD, Barer ML. Seniors' prescription drug cost inflation and cost containment: evidence from British Columbia. Health Policy 2004; 68:299-307.

16. Acurcio FA, Rozenfeld S, Ribeiro AQ, Klein CH, Moura CS, Andrade CR. Utilização de medicamentos por aposentados brasileiros. 1 - metodologia $\mathrm{e}$ resultados de cobertura de inquérito multicêntrico. Cad Saúde Pública 2006; 22:87-96.

17. Brasil. Lei $n^{\circ}$. 9.787. Altera a Lei $n^{\circ}$. 6.360, de 23 de setembro de 1976, que dispõe sobre a vigilância sanitária, estabelece o medicamento genérico, dispõe sobre a utilização de nomes genéricos em produtos farmacêuticos e dá outras providências. Diário Oficial da União 1999; 10 fev.

18. Agência Nacional de Vigilância Sanitária. Resolução ${ }^{\circ}$. 140. Estabelece regras das bulas de medicamentos para pacientes e para profissionais de saúde. Diário Oficial da União 2003; 29 mai.

19. Ministério da Saúde. Relação nacional de medicamentos essenciais - RENAME. Brasília: Ministério da Saúde; 2002.

20. World Health Organization. Report of the WHO Expert Committee on national drug policies. Geneva: World Health Organization; 1995.
21. Brasil. Lei no ${ }^{\circ}$ 10.742. Define normas de regulação para o setor farmacêutico, cria a Câmara de Regulação do Mercado de Medicamentos - CMED e altera a Lei $n^{\circ}$. 6.360, de 23 de setembro de 1976, e dá outras providências. Diário Oficial da União 2003; 7 out.

22. Secretaria Executiva da Câmara de Medicamentos.

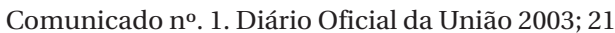
fev.

23. Brasil. Lei $n^{\circ}$. 10.699. Dispõe sobre o salário mínimo a partir de $1^{\circ}$ de abril de 2003, e dá outras providências. Diário Oficial da União 2003; 9 jul.

24. Grima DT, Burge RT, Becker DL, Tosteson AN. Short-term cost-effectiveness of bisphosphonate therapies for postmenopausal osteoporotic women at high risk of fracture. Pharmacy \& Therapeutics 2002; 27:448-55.

25. Forder J, Kavanagh S, Fenyo A. A comparison of the cost-effectiveness of sertraline versus tricyclic antidepressants in primary care. J Affect Disord 1996; 38:97-111.

26. Shukla VK, Otten N, editors. Drug treatments for Alzheimer's disease. III. A review of pharmacoeconomic evaluations. Ottawa: Canadian Coordinating Office for Health Technology Assessment; 2000.

27. Associação Brasileira das Indústrias de Medicamentos Genéricos - Pró-genéricos. Informações de mercado ano 2003. http://www.progenericos. org.br/info_mercado.shtml (acessado em 22/ $\mathrm{Jul} / 2005)$.

28. Associação Brasileira das Indústrias de Medicamentos Genéricos - Pró-Genéricos. Evolução do mercado de genéricos no Brasil - jun/04 a mai/05. http://www.progenericos.org.br/info_mercado. shtml (acessado em 22/Jul/2005).

29. Fox PD. Prescriptions drug benefits: cost management issues for Medicare. Health Care Financ Rev 2003; 25:7-21.

30. Laing R, Waning B, Gray A, Ford N, Hoen E. 25 years of the WHO essential medicines lists: progress and challenges. Lancet 2003; 361:1723-9.

31. Guerra Junior AA, Acurcio FA, Gomes CAP, Miralles M, Girardi SN, Werneck GAF, et al. Disponibilidade de medicamentos essenciais em duas regiões de Minas Gerais, Brasil. Rev Panam Salud Pública 2004; 15:168-75.

Recebido em 01/Fev/2006

Versão final reapresentada em 03/Ago/2006

Aprovado em 22/Dez/2006 